\title{
Antibacterial and Remineralization Efficacy of Casein Phosphopeptide, Glycomacropeptide Nanocomplex, and Probiotics in Experimental Toothpastes: An In Vitro Comparative Study
}

\author{
Hanaa Elgamily ${ }^{1}$ Engie Safwat ${ }^{1}$ Zainab Soliman ${ }^{2}$ Heba Salama $^{3}$ Hoda El-Sayed ${ }^{3}$ Mohamed Anwar ${ }^{2}$
}

\footnotetext{
${ }^{1}$ Oral and Dental Research Division, Restorative and Dental Materials Department, National Research Centre, Giza, Egypt

2Operative Dentistry Department, Faculty of Dentistry, Ain Shams University, Cairo, Egypt

${ }^{3}$ Division of Food Industries and Nutrition, Food Industries and Nutrition Division, Dairy Science Department, National Research Centre, Giza, Egypt
}

Eur J Dent 2019;13:391-398

\begin{abstract}
Address for correspondence Hanaa Elgamily, BDS, MSc, PhD, Operative Dentistry, Restorative and Dental Materials Department, National Research Centre, 33 El Bohouth st. (former El Tahrir st.), Dokki, Giza 12622, Egypt (e-mail: hanaa_elgamily@yahoo.com).
\end{abstract}

\begin{abstract}
Keywords

- casein

phosphopeptides

- amorphous calcium

phosphate

- probiotic

- glycomacropeptide

- anticariogenic

- remineralization

Objective This article evaluates the antibacterial and remineralization potential of experimentally prepared toothpastes containing different mixtures of nano casein phosphopeptides (nCPP), nano amorphous calcium phosphate (nACP), probiotic Lactobacillus rhamnosus B-445 (L. rhamnosus), and nano glycomacropeptide (nGMP).

Materials and Methods Five experimental toothpaste samples were prepared and grouped, such that group (A0) was the experimental toothpaste base formula. Groups (A1), (A2), (A3), and (A4) were the experimental toothpastes containing: nCPP; nCPP and nACP; nCPP, nACP, and L. rhamnosus, and nCPP, nACP, and nGMP, respectively. Group (A5) was the commercial group (GCMI Paste Plus). The five groups were screened against Streptococcus mutans (ATCC 25175) growth, and investigated for their remineralizing potentials on demineralized bovine enamel using Vickers microhardness test (Vickers hardness number [VHN]). Scanning electron microscope (SEM) images were obtained for the demineralized and remineralized enamel of the two most effective toothpastes against in vitro bacterial induced enamel demineralization. Statistical analysis was performed using one-way analysis of variance (ANOVA) as well as repeated measures ANOVA followed by Tukey's post hoc test.

Results Both (A3) and (A4) were significantly higher in mean inhibition zone diameters than group (A1) and (A2). Group (A4) showed the highest statistical significance in the mean difference between VHN values of demineralization and 15 days remineralization period. SEM images showed the deposition of nano-sized particles fill the microrough surface pattern of the etched enamel.

Conclusion All these findings suggest the use of probiotic, nCPP-nACP, and nGMP as a dental anticariogenic and remineralizing active agents.
\end{abstract}

\section{Introduction}

Dental caries is an incredibly widespread dental disease and is a first-rate public health problem. ${ }^{1} \mathrm{~A}$ carious lesion is initiated by specific bacteria in oral biofilm that is able to demineralize teeth under suitable environment inside the oral cavity. ${ }^{2}$ With clear understanding regarding the demineralization-remineralization theory of caries initiation, 
emphasis in present-day dentistry is on remineralization of carious lesion. ${ }^{3}$ In the modern era of minimal intervention dentistry, remineralizing agents in a wide variety of formulations have been developed to promote remineralization of the demineralized enamel., ${ }^{3,4}$

Fluoride is the oldest and most widely used remineralizing agent. Concerns regarding fluoride toxicity and its side effects induced the need to safer means for enamel remineralization. ${ }^{5}$ One of the novel remineralization methods is the utilization of calcium phosphate particles dissolved in casein milk protein, that creates amorphous calcium phosphate particles super saturation around the tooth, in the acidic environment of dental plaque, thus they are thought to enhance remineralization process. ${ }^{6,7}$ Casein milk protein is a cluster of protein molecules, and its technical name is casein phosphopeptide-amorphous calcium phosphate (CPP-ACP) nanocomplex, and is found to remineralize subsurface lesions in the human enamel. ${ }^{8}$ The CPP-ACP tooth remineralization mechanism of action is to provide a reservoir of free calcium and phosphate ions in the amorphous state within the plaque at the tooth surface, this reservoir maintains a supersaturation mineral state on the enamel surface. ${ }^{9}$ Commercial products such as MI Paste Plus (GC America) contains fluoride and CPP-ACP, and became available as a remineralizing treatment of white spot lesions and initial caries through maintaining calcium phosphate ions supersaturated in amorphous state on the enamel surface. However, fluoride has a slightly higher potential in the remineralization of early enamel caries lesions than that of CPP-ACP.,7

Glycomacropeptide (GMP) which is a hydrophilic part of casein, has attracted a great deal of attention due to its biological activities, which includes the reduction of dental caries through changing microbial composition of dental plaque from Streptococci to less cariogenic Actinomyces. ${ }^{10}$ Hence, formulating GMP into gum or toothpaste is a way to prevent caries and control dental plaque. ${ }^{11,12}$ As well, probiotic approach is a new preventive and therapeutic approach based on competition with oral pathogens for adhesion sites and nutrients or through restoring the microbial ecological balance in the oral cavity. ${ }^{13,14}$ Previous literatures revealed that long-term intake of Lactobacillus rhamnosus GG ATCC 53103 in milk which is a probiotic bacterium, had a beneficial effect on children's dental health, in addition to no evidence of an increase in plaque acidity. ${ }^{15,16}$

In a previous study, the preparation of nano-CPP (nCPP) from the tryptic digest of bovine casein and the preparation of nano-GMP (nGMP) by the action of chymosin on casein solution, were conducted. ${ }^{17}$ Through this study, the antiadhesive and antibacterial activities of four experimental solution containing different mixtures of nCPP, nano-ACP (nACP), probiotic L. rhamnosus, and nGMP were confirmed. Moreover, it was proved that $L$. rhamnosus had high stability in this solution for a practical period of the study time. Hence, the aim of this study is to prepare four experimental toothpastes containing different mixtures of probiotics, nCPP-nACP, and nGMP, and investigate their antibacterial and remineralizing potentials on demineralized enamel of bovine teeth in comparison to commercial product (GC MI Paste Plus). The null hypothesis was examined, first: that there is no difference in antibacterial efficacy between the experimental toothpastes and commercial one, and second: there is no difference in enamel microhardness between experimental toothpastes and commercial one.

\section{Materials and Methods}

\section{Preparation of Different Experimental Toothpastes}

Different mixtures from nCPP, nACP, L. rhamnosus $\left(10^{9} \mathrm{CFU} / \mathrm{g}\right.$ of $L$. rhamnosus B-445), and nGMP at $\mathrm{pH} 6.8$ were prepared according to the previous study. ${ }^{17}$ Each of these mixtures was incorporated in a specially prepared experimental toothpaste base formula which contained distilled water, thickener, surfactant, moist, flavor, and free sucrose sweetening agent in the concentration of 1:19 by weight (patent under registration no 2017/855, Ministry of Scientific Research, Academy of Scientific Research \& Technology, Egypt). A total of 100 samples were divided into 5 main groups $(n=20)$ : (A0), the experimental toothpaste base formula; (A1), nCPP experimental toothpaste; (A2), nCPP and nACP experimental toothpaste; (A3), nCPP, nACP, and probiotic strain L. rhamnosus B-445 ( $10^{9} \mathrm{CFU} / \mathrm{g}$ ) experimental toothpaste; (A4), nCPP, nACP, and nGMP; and (A5), commercial group (MI Paste Plus, $0.2 \%$ $\mathrm{NaF}$, Recaldent, GC Corporation, Tokyo, Japan). Each group was subdivided into two subgroups $(n=10)$ according to the testing procedure performed.

\section{Testing Procedure \\ Antibacterial Assessment}

\section{Test Bacteria}

A serotype (c) strain of Streptococcus mutans (S. mutans) ATCC 25175 that was isolated from carious dentine, and purchased from MIRCEN (Microbiological Resources Centre, Cairo, Egypt) was selected for susceptibility assay. $S$. mutans was streaked on tryptic soy agar (TSA; Difco Laboratories, Detroit, Michigan, United States) and incubated for 18 to 24 hours at $37^{\circ} \mathrm{C}$. An inoculum was prepared according to the Clinical and Laboratory Standards Institute by direct colony suspension method..$^{18}$ Bacterial colonies of an overnight culture of S. mutans were suspended in Mueller-Hinton Broth (CM0405, OXOID) and have been adjusted to 0.5 McFarland standards to reach a final inoculum corresponding to approximately $1 \times 10^{8} \mathrm{CFU} / \mathrm{mL}$.

\section{Agar Well Diffusion Assay}

The antimicrobial activity of the experimentally prepared toothpastes was screened against $S$. mutans growth by agar well diffusion assay. A $50 \mu \mathrm{L}$ of diluted $S$. mutans $\left(1 \times 10^{8} \mathrm{CFU} / \mathrm{mL}\right)$ were spread on the surface of TSA plate. A sterile $6-\mathrm{mm}$ cork borer was used to cut five wells at equidistance in the plate. Similar calculated amount (250 mg) of four experimental toothpastes and the commercial one was introduced each into the five wells. Ten replicate agar plates each containing five wells for the tested toothpastes were incubated at 35 to $37^{\circ} \mathrm{C}$ for 24 hours. At the end of the incubation period, the antimicrobial activity of the tested toothpastes was evaluated by measuring 10 inhibition zone diameters for each group (in mm). ${ }^{19}$ 


\section{Hardness Testing}

A total of 50 bovine incisors were analyzed in this study for surface hardness using Vickers microhardness tester (Shimadzu HMV-M Micro-hardness Tester; Newage Testing Instruments Inc., Southampton, Pennsylvania, United States) under $200 \mathrm{~g}$ load, and 15 seconds dwell time. ${ }^{20,21}$ The sample size was estimated for microhardness test to be 10 enamel specimens for each group considering a study power of $80 \%$ and statistical significance of $5 \%(a=0.05)$ based on a mean \pm standard deviation (SD) of calcium ion loss for triclosan and sodium fluoride toothpastes of $12.9 \pm 0.8$ and $11.3 \pm 0.3$, respectively, and 5 specimens per group. ${ }^{22}$ All the selected teeth were thoroughly cleaned under running tap water using soft toothbrush and the crowns were resected from the cement-enamel junction using a diamond fissure bur mounted on a high-speed handpiece (NSK, Japan)..23 All the crowns were fixed in autopolymerizing acrylic resin (Acropars, Marlic Medical Industries Co, Tehran, Iran) in a manner that the buccal surface of the enamel was faced upwards and was not covered by acrylic resin. The surface of the samples was ground to provide a flat surface and remove possible excess resin, using minimum hand pressure with silicone carbide grinding papers (Buehler-Met; Agar Scientific Limited, Cambridge, United Kingdom) of 300 to 2,000 grits in a progressive way under running water and polished using 1 $\mu \mathrm{m}$ diamond suspension. ${ }^{20}$ The mean value of the measured Vickers hardness number (VHN) of three indentations were obtained for each tooth specimen of the five groups at baseline before enamel treatment, then following a 90 -second exposure to phosphoric acid etching using Kerr gel etchant 37.5\% phosphoric acid to result in a minimum loss of surface enamel, exposure of more reactive enamel through exposing the enamel prism end structure, increasing the surface area of the enamel, and producing a certain degree of subsurface porosity for interaction with preventative and remineralizing agents. ${ }^{24,25}$ Subsequently, each tooth specimen of the five groups received remineralization treatment through uniform brushing once daily for 15 days. A 3-minute brushing process utilizing $1 \mathrm{~cm}^{3}$ of the respective paste through mild manual tooth brushing pressure such that the bristles were stabilized on the tooth surface. After brushing, the samples were rinsed with distilled water and then immersed in Fusayama Meyer's artificial saliva, composed of $\mathrm{NaCl}(0.400 \mathrm{~g} / \mathrm{L}), \mathrm{KCl}(0.400 \mathrm{~g} / \mathrm{L}), \mathrm{CaCl}_{2} \cdot \mathrm{H}_{2} \mathrm{O}(0.906 \mathrm{~g} / \mathrm{L})$, $\mathrm{NaH}_{2} \mathrm{PO}_{4} \cdot 2 \mathrm{H}_{2} \mathrm{O}(0.690 \mathrm{~g} / \mathrm{L}), \mathrm{Na}_{2} \mathrm{~S} \cdot 9 \mathrm{H}_{2} \mathrm{O}(0.005 \mathrm{~g} / \mathrm{L})$, and urea $(1 \mathrm{~g} / \mathrm{L})$ with a pH of 7.1 at room temperature. ${ }^{24}$ The artificial saliva solution was replenished every 24 hours. Meanwhile, the treated enamel teeth specimens were tested for surface hardness at day 7 and finally at day 15 . Results were expressed as the mean and SD of VHN values of each group sample at baseline, demineralization, and remineralization at day 7 and day 15 , as well as a comparison was made for the remineralization potential among different groups through calculating the mean difference between VHN values of demineralization and 15 days' remineralization period. ${ }^{26,27}$

\section{Scanning Electron Microscope Characterization}

Group samples that showed superior antimicrobial and microhardness values were selected for qualitative analysis using scanning electron microscope (SEM). SEM Model
Quanta 250 Field Emission Gun was attached with EDX Unit (Energy Dispersive X-ray Analyses), with accelerating voltage of $30 \mathrm{KV}$, and for SEM observation were selected at $1,000 \times$ and 2,000× magnification and resolution for Gun.1n (FEI Company, Netherlands). Images were obtained at baseline for intact enamel before demineralization, and then after acid etching demineralization, finally third image was obtained after 15 days' remineralization period.

\section{Statistical Analysis}

Numerical data were explored for normality by checking the distribution of data, calculating the mean and median values, as well as using the tests of normality (Kolmogorov-Smirnov and Shapiro-Wilk tests). All data showed parametric distribution and were presented as mean and SD values. One-way analysis of variance (ANOVA) followed by Tukey's post hoc test was used to compare between more than two groups. Repeated measures ANOVA followed by Tukey's post hoc test was used to study the changes by time within each group. The significance level was set at $p \leq 0.05$. Statistical analysis was performed with IBM SPSS Statistics Version 20 for Windows.

\section{Results}

\section{Mean Inhibition Zones Diameters Exhibited by Different Toothpastes}

As shown in - Table 1, all nCPP containing experimental toothpastes (A1), (A2), (A3), and (A4) showed an antibacterial potential against $S$. mutans growth. Also, there was no inhibition zone diameter in the groups of the experimental toothpaste base (A0) and the commercial product (A5). As regards the group (A3) and (A4) samples, there was no statistically significant difference among them, and both were significantly higher in mean inhibition zone diameters than group (A1) and (A2) samples. Similarly, there was no statistically significant difference between groups (A1) and (A2).

\section{The Microhardness Results}

The VHN values at baseline, before demineralization, were decreased considerably following demineralization for all groups as revealed in - Table 2 . There was no statistically significant increase in the VHN values for groups (A0) and (A1) after 15 days' remineralization periods than the demineralization VHN values. As regards groups (A2) and (A3), there was a statistically significant increase in VHN values after 7 days' remineralization periods but there was no statistically difference after 15 days' remineralization periods. On the opposite, group (A4) showed no significant increase in VHN at day 7 remineralization period, but there was a high significant increase after 15 days of remineralization. For the commercial group (A5), there was a significant gradual increase in VHN at day 7 and 15 remineralization.

Concerning the comparison of remineralization potential between different groups as represented by the amount of increase of VHN values obtained after calculating the mean difference between VHN values of demineralization and 15 days' remineralization period within each group, results revealed that the highest statistically significant values was 
Table 1 The mean, standard deviation (SD) values of overall inhibition zone results for all groups

\begin{tabular}{|l|l|l|}
\hline \multirow{2}{*}{ Variables } & \multicolumn{2}{|l|}{$\begin{array}{l}\text { Overall Inhibition zone diameter } \\
(\mathrm{mm})\end{array}$} \\
\cline { 2 - 3 } & Mean & $\begin{array}{l}\text { Standard } \\
\text { deviation }\end{array}$ \\
\hline A0 (base) & 0.00 & 0.00 \\
\hline A1 (CPP) & $8.10^{\text {b. }}$ & 2.7 \\
\hline A2 (CPP/ACP) & $10.4^{\text {b }}$ & 2.7 \\
\hline $\begin{array}{l}\text { A3 (CPP/ACP/ } \\
\text { Probiotic) }\end{array}$ & $17.1^{\text {a }}$ & 1.9 \\
\hline $\begin{array}{l}\text { A4 (CPP/ACP/ } \\
\text { GMP) }\end{array}$ & $15.9^{\text {a }}$ & 2.2 \\
\hline A5 (Commercial) & 0.00 & 0.00 \\
\hline P-Value & $\leq 0.001^{*}$ \\
\hline
\end{tabular}

Abbreviations: ACP, amorphous calcium phosphate; CPP, casein phosphopeptide; GMP, glycomacropeptide.

Note: Superscripts with different small letters indicate statistically significance difference within the same column.

*Significant $(p \leq 0.05)$; nonsignificant $(p>0.05)$. to excessive roughening and surface irregularities after acid etching as revealed in - Figs. 2B and 3B. After remineralization using group (A3) and (A4) samples, the remineralized enamel exhibited precipitation of white nano-sized particles filling the microrough surface pattern of the etched enamel after 15 days' remineralization treatment, these white nanoparticles were few in (A3) samples and numerous in (A4) samples as revealed in - Figs. 2C, D and 3C, D.

\section{Discussion}

Based on the current results, all experimental toothpastes containing nCPP showed significantly higher antimicrobial effect compared with the commercial group containing MI paste (A5). This finding could be attributed to the presence of CPP in the nano form which enhanced its antimicrobial capacity expressed as inhibition zone for S. mutans. ${ }^{17}$ Even though casein milk protein has wide range of significant benefits as an anticariogenic additive to food, toothpaste, or ingesting water, its use has not been applied due to its negative organoleptic characteristics and the massive quantity required for efficacy. ${ }^{728}$ The antibacterial potential of experimental

Table 2 The mean, standard deviation (SD) of microhardness in different groups

\begin{tabular}{|c|c|c|c|c|c|c|c|c|c|}
\hline \multirow{3}{*}{ Variables } & \multicolumn{9}{|c|}{ VHN } \\
\hline & \multicolumn{2}{|l|}{ Initial } & \multicolumn{2}{|c|}{$\begin{array}{l}\text { After } \\
\text { demineralization }\end{array}$} & \multicolumn{2}{|c|}{$\begin{array}{l}\text { After treatment } \\
7 \mathrm{~d}\end{array}$} & \multicolumn{2}{|c|}{$\begin{array}{l}\text { After treatment } \\
15 \mathrm{~d}\end{array}$} & \multirow[t]{2}{*}{$p$-Value } \\
\hline & Mean & SD & Mean & SD & Mean & SD & Mean & SD & \\
\hline A0 (base) & $177.7^{\mathrm{Aa}}$ & 37.4 & $63.3^{\mathrm{Ab}}$ & 20.7 & $64.4^{\mathrm{Ab}}$ & 16.6 & $58.1^{\mathrm{Ab}}$ & 16.6 & $<0.001^{*}$ \\
\hline $\mathrm{A} 1$ (CPP) & $182.3^{\text {Aa }}$ & 46.9 & $41.5^{\mathrm{Ab}}$ & 13.5 & $83.4^{\mathrm{Bb}}$ & 18.4 & $78.4^{\mathrm{Ab}}$ & 21.1 & $<0.001^{*}$ \\
\hline $\mathrm{A} 2$ (CPP/ACP) & $195.9^{\text {Aa }}$ & 49.2 & $48.4^{\mathrm{Ac}}$ & 18.8 & $100.9^{\mathrm{Bb}}$ & 16.5 & $124.5^{\mathrm{Bb}}$ & 16.05 & $<0.001^{*}$ \\
\hline A3 (CPP/ACP/Probiotic) & $253.2^{\mathrm{Ba}}$ & 30.9 & $63.9^{\mathrm{AC}}$ & 19.7 & $117.4^{\mathrm{cb}}$ & 12.9 & $125.8^{\mathrm{Bb}}$ & 18.8 & $<0.001^{*}$ \\
\hline A4 (CPP/ACP/GMP) & $276.6^{\mathrm{Ba}}$ & 42.9 & $54.7^{\mathrm{Ac}}$ & 15.1 & $81.5^{\mathrm{BC}}$ & 14.5 & $149.9^{\mathrm{cb}}$ & 13.2 & $0.0032^{*}$ \\
\hline A5 (Commercial) & $265.3^{\text {Ba }}$ & 34.9 & $47.2^{\mathrm{Ad}}$ & 13.4 & $85.7^{\mathrm{BC}}$ & 8.4 & $130.6^{\mathrm{Bb}}$ & 17.1 & $<0.001^{*}$ \\
\hline$p$-Value & \multicolumn{2}{|l|}{$0.01^{*}$} & \multicolumn{2}{|l|}{$0.09 \mathrm{~ns}$} & \multicolumn{2}{|c|}{$<0.013^{*}$} & \multicolumn{2}{|l|}{$0.008^{*}$} & \\
\hline
\end{tabular}

Abbreviations: ACP, amorphous calcium phosphate; CPP, casein phosphopeptide; GMP, glycomacropeptide; VHN, Vickers hardness number. Note: Means followed by different letters (lowercase horizontally and uppercase vertically) indicate statistically significant differences ( ${ }^{*}$ significant $[p<0.05]$ and nonsignificant $[\mathrm{ns}][p>0.05])$.

for group (A4) (112.43 \pm 39.3) followed by the commercial group (A5) (94.95 \pm 25.1 ) and group (A2) (77.43 \pm 27.3 ); both had no statistically significant difference. The lowest mean difference was for the groups (A1) and (A3) that showed $36.19 \pm 11.2$ and $52.23 \pm 13.4$, respectively, with no statistical difference between them. Finally, the lowest mean difference value was found for the group (A0) $(2.75 \pm 2.2)$ (-Fig. 1).

\section{SEM Analysis of the Enamel Surface}

SEM images were analyzed for the characterization of the enamel samples treated with the two most effective toothpastes of group (A3) and (A4) against in vitro bacterial induced enamel demineralization. SEM image investigation showed smooth and intact enamel surface of the baseline normal bovine enamel sample before demineralization as shown in -Figs. 2A and 3A. This intact enamel was converted toothpaste containing $\mathrm{CPP}-\mathrm{ACP}$ in group (A2) related to CPP-ACP has a well-known ability to inhibit the adhesion of cariogenic Streptococci to the tooth surface that alters the plaque nature to noncariogenic plaque. ${ }^{29}$ On the other hand, the antimicrobial capacity of the experimental toothpaste in group (A3) was rather enhanced by the addition of probiotic L. rhamnosus with nano casein glycoprotein due to competition for nutrients in agar medium between the pathogen and the probiotic strain, or production of antimicrobial substances that can inhibit growth of the pathogen. ${ }^{30,31}$ The latter results are in agreement with Saha et $\mathrm{al}^{32}$ and Stamatova and Meurman ${ }^{33}$ who demonstrated that the beneficial role of probiotics is particularly based totally on their antagonistic effect on pathogens. The inhibitory activity in group (A4) could be related to the incorporation of GMP into nano-CPP experimental toothpaste. The superior advantage of GMP 


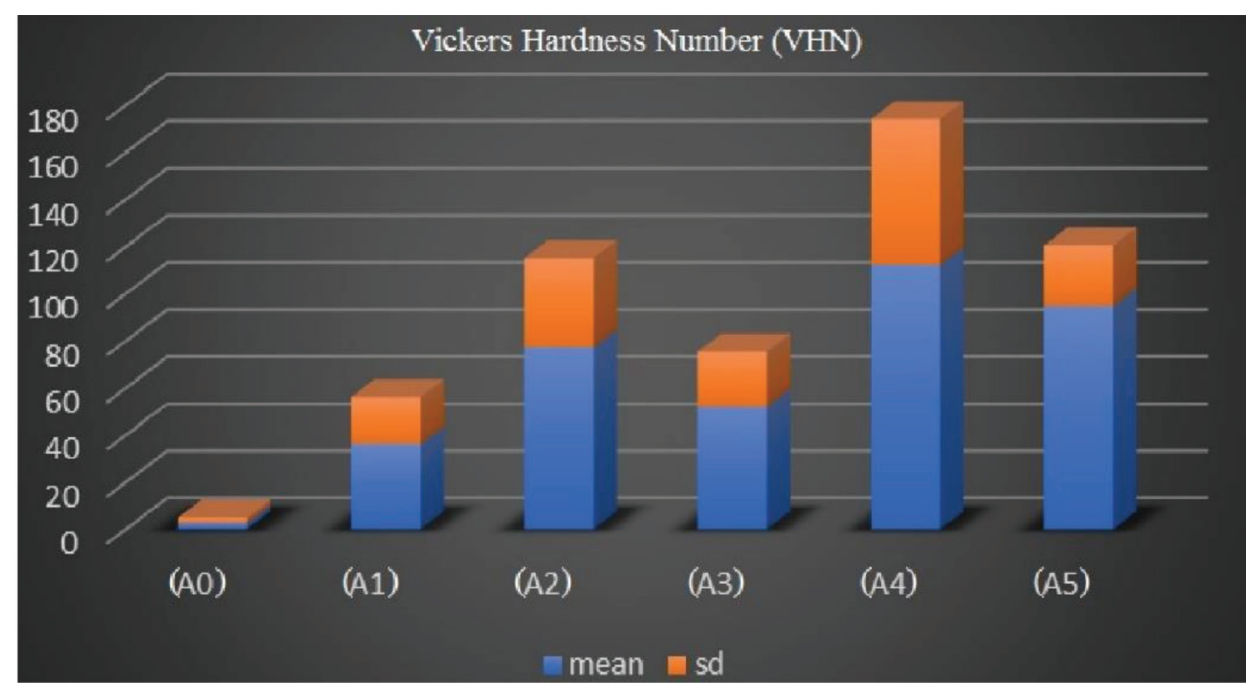

Fig. 1 Bar chart representing the amount of increase in the Vickers hardness number (VHN) values after 15 days' remineralization period.
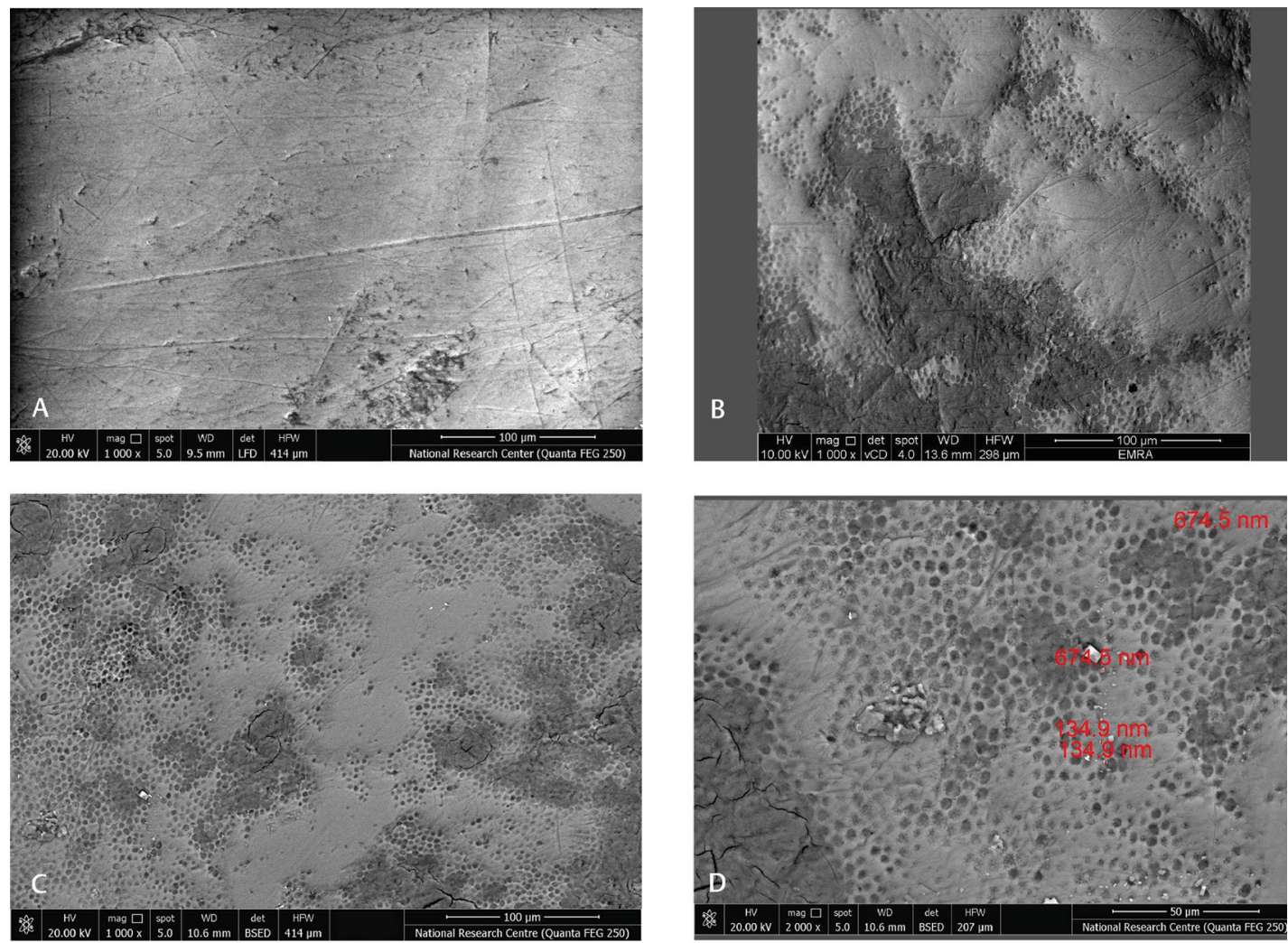

Fig. 2 (A) The baseline of normal bovine enamel sample. (B) Demineralized enamel. (C) Remineralized enamel exhibiting precipitation of a few numbers of white nanoparticles on the dentinal tubules orifices after 15 days' treatment using (A3) toothpaste at magnification (1, 000×). (D) Remineralized enamel exhibiting precipitation of a few numbers of white nanoparticles on the dentinal tubules orifices after 15 days' treatment using (A3) toothpaste at magnification (2,000x). 

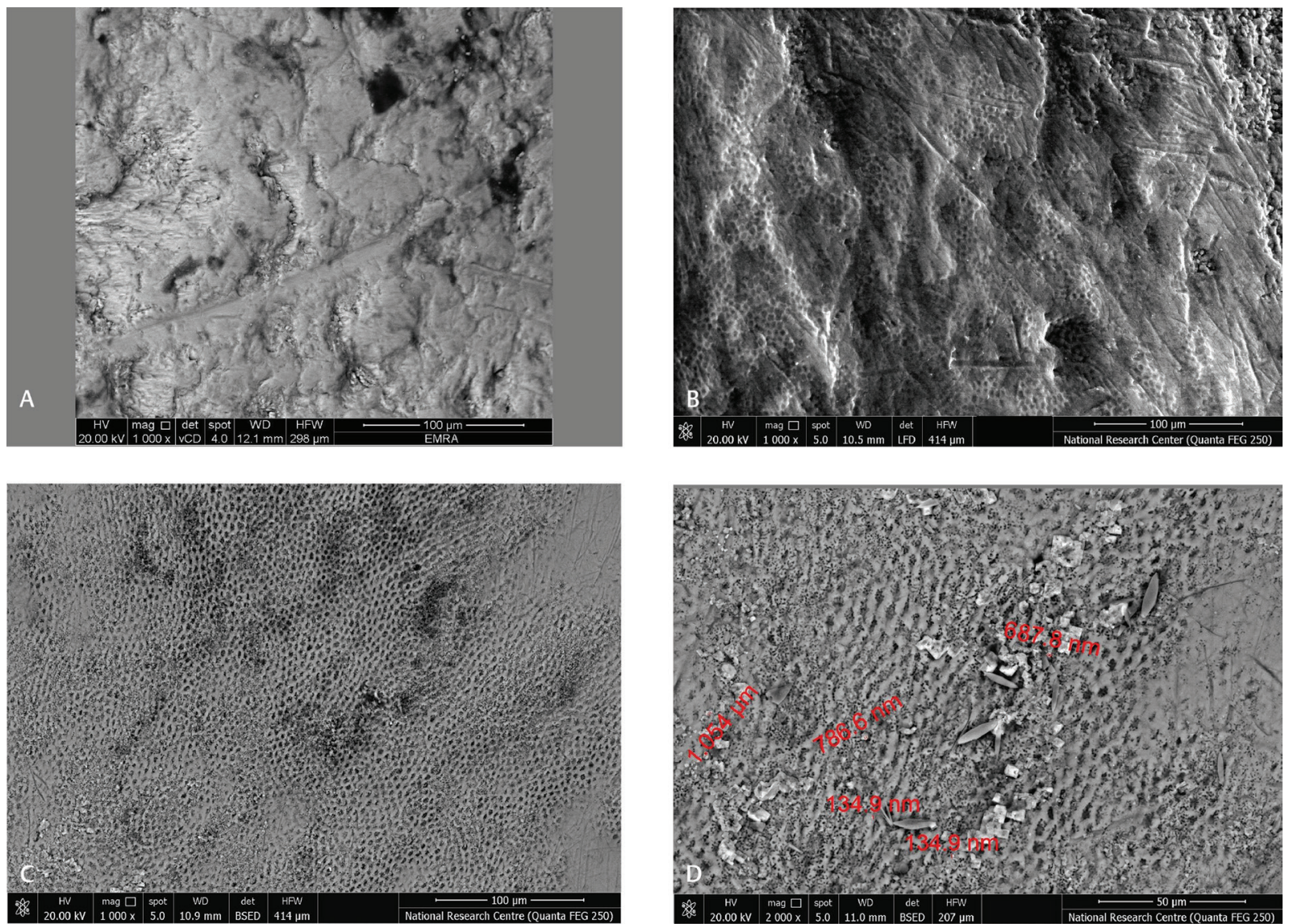

Fig. 3 (A) The baseline of normal bovine enamel sample. (B) Demineralized enamel. (C). Remineralized enamel exhibiting precipitation of multiple numbers of white nanoparticles on the dentinal tubules orifices after 15 days' treatment using (A4) toothpaste at magnification (1,000×). (D) Remineralized enamel exhibiting precipitation of multiple numbers of white nanoparticles on the dentinal tubules orifices after 15 days' treatment using (A4) toothpaste at magnification (2,000×).

is its highly negative charge at low pH while the other milk proteins derivatives are positively charged, and this allows the GMP adsorption on the anionic cariogenic bacterial cell wall while the other proteins (CCP-ACP) containing toothpaste in the group (A4) are washed free. ${ }^{34,35}$ It may be possible that GMP blocked the adhesins receptor which is a part of the bacterial colonization process as reported by Neeser et al. ${ }^{36}$ Consequently, this could negatively affect the ability of cariogenic S. mutans for colonization, thereby GMP could be considered as a bacteriostatic agent. However, due to the fact that tested teeth samples were totally free of dental plaque and calculus due to the previous teeth preparation and demineralization step, it is impossible to check the full capability of GMP. As reported by Neeser et a ${ }^{36}$ and Schüpbach et $\mathrm{al}^{37} \mathrm{GMP}$ has the ability to inhibit the adhesion of cariogenic bacteria $S$. mutans to oral surfaces.

The present average VHN results for bovine teeth was found to be within the 177 to 265 range which is in agreement with previous studies. ${ }^{2,38}$ At both remineralizing periods ( 7 and 15 days), The MI paste (A5) showed higher microhardness results than the control (A0) at both experimental time intervals. The results differed from other studies that compared MI paste with other commercial toothpastes due to the remineralizing effect of fluoride present in other toothpastes. ${ }^{39}$ Upon adding CPP to the experimental toothpaste, the microhardness (A1) results at the 7 days were similar to the MI paste, though the ACP containing MI paste showed statistically higher microhardness at 15 days, and this could be attributed to the presence of the ACP in the MI paste which is reported to be responsible for enamel remineralization. ${ }^{40}$ With the addition of ACP to the experimental toothpaste (A2), the microhardness was significantly higher than MI paste at 7 days but both groups showed similar results at 15 days. This difference in microhardness at 7 days could be attributed to the presence of the CPP in a nanostructure in the experimental toothpaste which fastened its remineralizing capacity in comparison to the MI paste. The addition of probiotic bacteria in the toothpaste (A3) resulted in statistically higher microhardness at the 7 days with obvious decline at 15 days' period to give the lowest hardness results in all groups having ACP. This decline in the microhardness could be attributed to the nature of the L. rhamnosus bacteria which is a phosphate accumulating bacteria that tends to accumulate polyphosphates resulting in the decline of phosphate in the surrounding which can lead to undersaturated conditions relative to the hydroxyapatite which might lead to demineralization potentials in dental plaque and lesser degrees of remineralization in the conducted study. ${ }^{41}$ Though the inhibitory effect of group (A3) toothpaste containing probiotic against $S$. mutans growth was in accordance with many 
studies, ${ }^{42,43}$ the remineralization capacity of this group needs prolonged studies in addition to in vivo studies to observe the actual effect of this toothpaste in oral conditions. Regarding the nGMP group (A4), the highest microhardness remineralization results were reported for this group. The reason behind it might be the synergistic effect between nGMP with nCPP in the experimental toothpaste that could be released and coated the hydroxyapatite particles to suppress demineralization and to enhance remineralization during an acid attack. ${ }^{44}$ Accordingly, the combination of the microhardness results with bacterial inhibition zone shows the ability of (A4) group to satisfy both needs for caries management.

The outcomes suggested in this study are based totally upon an in vitro version, which has numerous innate obstacles. Full remineralizing environment was created to check the experimental toothpastes and commercial MI paste's ability to remineralize a $37.5 \%$ phosphoric acid demineralized tooth, without any further demineralization occurring. Hence, this study lacked a demineralization-remineralization cycling model, leading to create an environment that might never naturally exist inside the oral cavity. The exclusion of the demineralization inside the study ought to limit the mechanism of action of CPP-ACP containing toothpastes given that an acidic environment is needed to initiate the active component in the past. ${ }^{45,46}$ As a result, none of the remineralizing toothpastes used in this study, as well as the commercial MI paste, was able to restore the microhardness to its original values; however, their antimicrobial property put them in a definite advantage compared with the MI paste. Hence, the null hypothesis was rejected.

\section{Conclusion}

Under the limitation of the following study, the two experimental toothpastes containing nCPP-nACP with probiotic strain or with nGMP proved a potential remineralizing property with a more promising antimicrobial efficiency. Longer time intervals are needed to study the effect of them on demineralized enamel; in addition, in vivo studies are required to determine their effect on oral flora. The selected $L$. rhamnosus probiotic strain represents a new concept in the oral hygiene measurements and could be recommended as a therapeutic agent in toothpastes as there is no issue of antibiotic resistance.

\section{Conflict of Interest}

Dr. Salama reports a patent under registration no 2017/855 pending. Dr. Safwat reports a patent under registration no 2017/855 pending. Dr. Elgamily reports a patent under registration no 2017/855 pending. Dr. Samir reports a patent under registration no 2017/855 pending.

\section{References}

1 Pradeep K, Rao PK. Remineralizing agents in the non-invasive treatment of early carious lesions. Int J Dent Case Rep. 2011;1:73-84

2 Sharma A, Rao A, Shenoy R, Suprabha BS. Comparative evaluation of nano-hydroxyapatite and casein phosphopeptide-amorphous calcium phosphate on the remineralization potential of early enamel lesions: an in vitro. study. J Orofac Sci 2017;9:28-33

3 Walsh LJ. Contemporary technologies for remineralization therapies: a review. Int Dent SA. 2009;11:6-16

4 Zero DT. Dentifrices, mouthwashes, and remineralization/caries arrestment strategies. BMC Oral Health 2006;6(Suppl 1):S9

5 Kanduti D, Sterbenk P, Artnik B. Fluoride: a review of use and effects on health. Mater Sociomed 2016;28(2):133-137

6 Lata S, Varghese NO, Varughese JM. Remineralization potential of fluoride and amorphous calcium phosphate-casein phospho peptide on enamel lesions: an in vitro comparative evaluation. J Conserv Dent 2010;13(1):42-46

7 Reynolds EC. Anticariogenic complexes of amorphous calcium phosphate stabilized by casein phosphopeptides: a review. Spec Care Dentist 1998;18(1):8-16

8 Kumar VL, Itthagarun A, King NM. The effect of casein phosphopeptide-amorphous calcium phosphate on remineralization of artificial caries-like lesions: an in vitro study. Aust Dent J 2008;53(1):34-40

9 Reynolds EC. Inventor. Anticariogenic phosphopeptides. US Patent 5015628; May 14, 1991

10 Neeser JR, Golliard M, Woltz A, Rouvet M, Dillmann ML Guggenheim B. In vitro modulation of oral bacterial adhesion to saliva-coated hydroxyapatite beads by milk casein derivatives. Oral Microbiol Immunol 1994;9(4):193-201

11 Neeser JR. Inventor. Anti-plaque and anticaries agent. United States Patent US 4992420; 1991

12 Neeser JR. Inventor. Anti-plaque and anticaries agent. United States Patent US 4994441; 1991

13 Marsh PD. The commensal microbiota and the development of human disease - an introduction. J Oral Microbiol 2015;7:29128

14 Marsh PD, Head DA, Devine DA. Ecological approaches to oral biofilms: control without killing. Caries Res 2015; 49(Suppl 1) :46-54

15 Näse L, Hatakka K, Savilahti E, et al. Effect of long-term consumption of a probiotic bacterium, Lactobacillus rhamnosus GG, in milk on dental caries and caries risk in children. Caries Res 2001;35(6):412-420

16 Keller MK, Twetman S. Acid production in dental plaque after exposure to probiotic bacteria. BMC Oral Health 2012;12:44

17 Elgamily HM, Salama H, El-Sayed H, Safwat E, Abd El-Salam M. The promising efficacy of probiotics, casein phosphopeptide and casein macropeptide as dental anticariogenic and remineralizing agents part I: an in vitro study. Annu Res Rev Biol 2018;22(6):1-11

18 Clinical and Laboratory Standards Institute (CLSI) supplement M100S. Performance Standards for Antimicrobial Susceptibility Testing. 26th ed. Wayne, PA; 2016

19 Valgas C, De Souza SM, Smânia EFA, et al. Screening methods to determine antibacterial activity of natural products. Braz J Microbiol 2007;38:369, -3-11

20 Chuenarrom C, Benjakul P, Daosodsai P. Microhardness tests for enamel and dentin. Mater Res 2009;12:473-476

21 Asaizumi M, Karlinsey R, Mackey A, Kato T, Kuga T. In vitro. assessment of white-spot lesions treated with NaF plus tricalcium Phosphate (TCP) toothpastes using microtomography (micro-CT) J Dent Oral Hyg 2013;5:68-76

22 van Loveren C, Buijs JF, ten Cate JM; van C. The effect of triclosan toothpaste on enamel demineralization in a bacterial demineralization model. J Antimicrob Chemother 2000;45(2):153-158

23 Salem-Milani A, Zand V, Asghari-Jafarabadi M, Zakeri-Milani $\mathrm{P}$, Banifatemeh A. The effect of protocol for disinfection of extracted teeth recommended by Center for Disease Control (CDC) on microhardness of enamel and dentin. J Clin Exp Dent 2015;7(5):e552-e556 
24 Ahrari F, Poosti M, Motahari P. Enamel resistance to demineralization following Er:YAG laser etching for bonding orthodontic brackets. Dent Res J (Isfahan) 2012;9(4):472-477

25 Flaitz CM, Hicks MJ. Role of the acid-etch technique in remineralization of caries-like lesions of enamel: a polarized light and scanning electron microscopic study. ASDC J Dent Child 1994;61(1):21-28

26 Lin J, Han S, Zhu J, et al. Influence of fluoride-containing acidic artificial saliva on the mechanical properties of Nickel-Titanium orthodontics wires. Indian. J Dent Res 2012;23(5):591-595

27 Nagi SM, El Hoshy AZ, Elenin KA. Effect of two remineralizing agents on the microhardness and ultramorphology of demineralized enamel. Medicine Sciences and Healthcare Journal. $2015 ; 10(12): 2-12$

28 Hora B, Kumar A, Bansal R, Bansal M, Khosla T, Garg A. Influence of McInnes bleaching agent on hardness of enamel and the effect of remineralizing gel GC tooth mousse on bleached enamel-an. in vitro. study. Ind J Dent Sci. 2012;4:13-19

29 Arruda AO, Behnan SM, Richter A, White-spot lesions in orthodontics: incidence and prevention. In: Ming-Yu Li, ed. Contemporary Approach to Dental Caries. In Tech Europe; 2012 314-323

30 Reynolds EC. Advances in enamel remineralization: casein phosphopeptide-amorphous calcium phosphate. J Clin Dent 1999;10:86-88

31 Söderling EM, Marttinen AM, Haukioja AL. Probiotic lactobacilli interfere with Streptococcus mutans biofilm formation in vitro. Curr Microbiol 2011;62(2):618-622

32 Saha S, Tomaro-Duchesneau C, Malhotra M, Tabrizian M, Prakash S. Suppression of Streptococcus mutans and Candida albicans by probiotics: an in vitro study. Dentistry 2012;2(6):141-148

33 Stamatova I, Meurman JH. Probiotics: health benefits in the mouth. Am J Dent 2009;22(6):329-338

34 Kawasaki Y, Dosako S, Shimatani M, Idota T. Process for producing k-casein glycomacropeptides. United States Patent 5280107; 1994

35 Brody EP. Biological activities of bovine glycomacropeptide. Br J Nutr 2000;84(Suppl 1):S39-S46

36 Neeser JR, Chambaz A, Del Vedovo S, Prigent MJ, Guggenheim B. Specific and nonspecific inhibition of adhesion of oral actinomyces and streptococci to erythrocytes and polystyrene by caseinoglycopeptide derivatives. Infect Immun 1988a;56(12):3201-3208

37 Schüpbach P, Neeser JR, Golliard M, Rouvet M, Guggenheim B. Incorporation of caseinoglycomacropeptide and caseinophosphopeptide into the salivary pellicle inhibits adherence of mutans streptococci. J Dent Res 1996;75(10):1779-1788

38 Zanet CG, Fava M, Alves LAC. In vitro evaluation of the microhardness of bovine enamel exposed to acid solutions after bleaching. Braz Oral Res 2011;25(6):562-567

39 Oliveira P, Fonseca A, Silva EM, Coutinho T, Tostes MA. Remineralizing potential of CPP-ACP creams with and without fluoride in artificial enamel lesions. Aust Dent J 2016;61(1):45-52

40 Reynolds EC, Cai F, Shen P, Walker GD. Retention in plaque and remineralization of enamel lesions by various forms of calcium in a mouthrinse or sugar-free chewing gum. J Dent Res 2003;82(3):206-211

41 Breiland AA, Flood BE, Nikrad J, et al. Polyphosphate-accumulating bacteria: potential contributors to mineral dissolution in the oral cavity. Appl Environ Microbiol 2018;84(7):e02440-e17

42 Elgamily HM, Nagi SM, Kassem AA, Zaazou MH, Mehanna NS. In-vitro comparative study of antimicrobial activity of two plant extracts and probiotic strain against isolated oral cariogenic pathogen. RJPBCS 2015;6(3):1705-1709

43 Elgamily H, Mosallam O, El-Sayed H. Mosallam R. Antibacterial effectiveness of probiotic-based experimental mouthwash against cariogenic pathogen: an in vitro study. Eur J Dent 2018;12(1):7-14

44 Nejad AS, Kanekanian A, Tatham A. The inhibitory effect of glycomacropeptide on dental erosion. Dairy Sci Technol 2009;89:233-236

45 Azarpazhooh A, Limeback H. Clinical efficacy of casein derivatives: a systematic review of the literature. J Am Dent Assoc 2008;139(7):915-924, quiz: 994-995

46 Pulido MT, Wefel JS, Hernandez MM, et al. The inhibitory effect of MI paste, fluoride and a combination of both on the progression of artificial caries-like lesions in enamel. Oper Dent 2008;33(5):550-555 